\title{
Large Solid Pseudopapillary Tumor Pancreas with Extensive Splenoportal Thrombosis and Malignant Transformation-A Rare Entity
}

\author{
Thara Pratap ${ }^{1}$ Dhanya Jacob ${ }^{1}$ Abhishek Yadav ${ }^{2}$ \\ ${ }^{1}$ Department of Radiology, VPS Lakeshore Hospital, Kochi, Kerala, \\ India \\ ${ }^{2}$ Comprehensive Liver Care Institute, VPS Lakeshore Hospital, Kochi, \\ Kerala, India \\ ${ }^{3}$ Department of Internal Medicine and Rheumatology, VPS Lakeshore \\ Hospital, Kochi, Kerala, India \\ ${ }^{4}$ Department of Pathology, VPS Lakeshore Hospital, Kochi, Kerala, \\ India
}

\author{
Muhammed Jasim Abdul Jalal ${ }^{3} \quad$ Iona Leekha Mathew ${ }^{4}$
}

J Gastrointestinal Abdominal Radiol ISGAR 2022;5:196-201.

\begin{abstract}
Address for correspondence Muhammed Jasim Abdul Jalal, MBBS, DNB, MNAMS, MRCGP, MRCP, Department of Internal Medicine and Rheumatology, VPS Lakeshore Hospital, Nettoor.P.O., Maradu, NH 47Byepass, Kochi 682040, Kerala, India (e-mail: jasimabduljalal@yahoo.com).
\end{abstract}

\author{
Abstract \\ Keywords \\ - solid pseudopapillary \\ tumor \\ - papillary epithelial \\ neoplasm \\ - papillary cystic \\ neoplasm \\ - solid and papillary \\ neoplasm \\ - low-grade papillary \\ neoplasm \\ - Frantz or Hamoudi \\ tumor
}

Solid pseudopapillary tumor (SPT) is an uncommon low-grade malignant cystic exocrine neoplasm of the pancreas that typically affects young women. The tumor has an excellent prognosis after surgical resection. Vascular involvement is usually not seen at presentation. Local recurrence and metastasis are also rare. Herein, we present a case of large, locally advanced SPT in a young female with splenoportal extension and malignant transformation.

\section{Introduction}

Solid pseudopapillary tumor (SPT) is a rare pancreatic neoplasm occurring in females in the second and third decades. The mean age of presentation is 24 years and one-third occur in adolescents. ${ }^{1}$ It is also known as papillary epithelial neoplasm, papillary cystic neoplasm, solid and papillary neoplasm, low-grade papillary neoplasm, and Frantz or
Hamoudi tumor. ${ }^{2}$ The first published description of a solid pseudopapillary neoplasm (SPN) was by Frantz in 1959. Hamoudi and colleagues detailed the electron microscopic appearance of the tumor later.

The tumor often presents with abdominal pain or as a palpable mass. SPT is a potentially malignant lesion. ${ }^{3}$ Surgery is often curative, and the 5 -year survival is 95 to $97 \% .^{4,5}$ However, SPT has an unpredictable behavior and metastasis published online February 17, 2022
DOI https://doi.org/ $10.1055 / \mathrm{s}-0042-1742679$. ISSN 2581-9933.

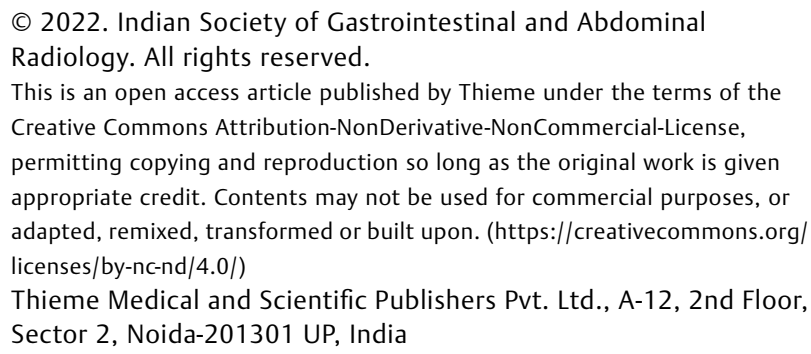


or local invasion has been reported in up to 10 to $15 \%$ of cases. ${ }^{4}$ Prognosis is poor when the tumor occurs in male patients, when there is local invasion into adjacent structures, vascular infiltration, cellular atypia, or capsular invasion on histopathology, and when it is unresectable or is metastatic tumor. ${ }^{2}$

\section{Case Report}

A 17-year-old female patient presented with abdominal distension, intermittent abdominal pain, loss of appetite, occasional episodes of vomiting, and weight loss of 3 months duration. She also had an episode of hematemesis and melena.

On clinical examination, the patient was icteric and the abdomen was distended. A mass was felt in the right hypochondrium and epigastric region.

Laboratory parameters were as follows: hemoglobin 8.4; total leukocyte count $4,290^{\wedge} 3 / \mu \mathrm{L}$; platelet count $1,90,000^{\wedge} 3$ / $\mu \mathrm{L}$; bilirubin total/direct $3.6 / 0.6 \mathrm{mg} / \mathrm{dL}$; aspartate aminotransferase $25 \mathrm{U} / \mathrm{L}$; alanine aminotransferase $24 \mathrm{U} / \mathrm{L}$; and alkaline phosphatase $102 \mathrm{U} / \mathrm{L}$.

Ultrasound showed a lobulated exophytic mass in the region of pancreatic head extending into the main portal vein and its branches with luminal expansion (-Fig. 1).

Contrast-enhanced computed tomography (CECT) was done for a better characterization of the lesion, which showed a large lobulated mass measuring $22 \times 19 \times 18 \mathrm{~cm}$ involving the head and body of pancreas with exophytic extension, displacing and compressing the adjacent bowel loops. There was no atrophy or duct dilatation of distal pancreas. A plain CT scan showed curvilinear calcification in the periphery of mass (-Figs. 2-4). Post-contrast study showed heterogeneous enhancement with tumor vascularity from branches of the superior mesenteric artery. There was no significant necrosis or cystic changes. Contiguous intravascular tumor extension was noted with tumor expanding the main portal vein to almost three times its size and into the entire intrahepatic portal vein radicles. Distally there was extension to the splenic vein up to the splenic hilum and inferiorly to the superior mesenteric vein for a length of approximately $6 \mathrm{~cm}$. Dilated intra-tumoral with peritumoral venous collaterals were noted (-Fig. 5) with thrombosis of a few collaterals in the mesentery and omentum. There was associated mild intrahepatic biliary radicle dilatation. Minimal free fluid was present. The possible differentials considered were SPT, neuroendocrine tumor, and leiomyoma/leiomyosarcoma as these are the tumors which present with intravascular extension.

Ultrasound-guided Trucut biopsy from the tumor was suggestive of SPN. Radiological features favored a locally aggressive SPEN in view of intravenous extension; however, the biopsy had not revealed any features of malignancy.

The patient was taken up for surgery, as the tumor was locally advanced and portal hypertension as evidenced by
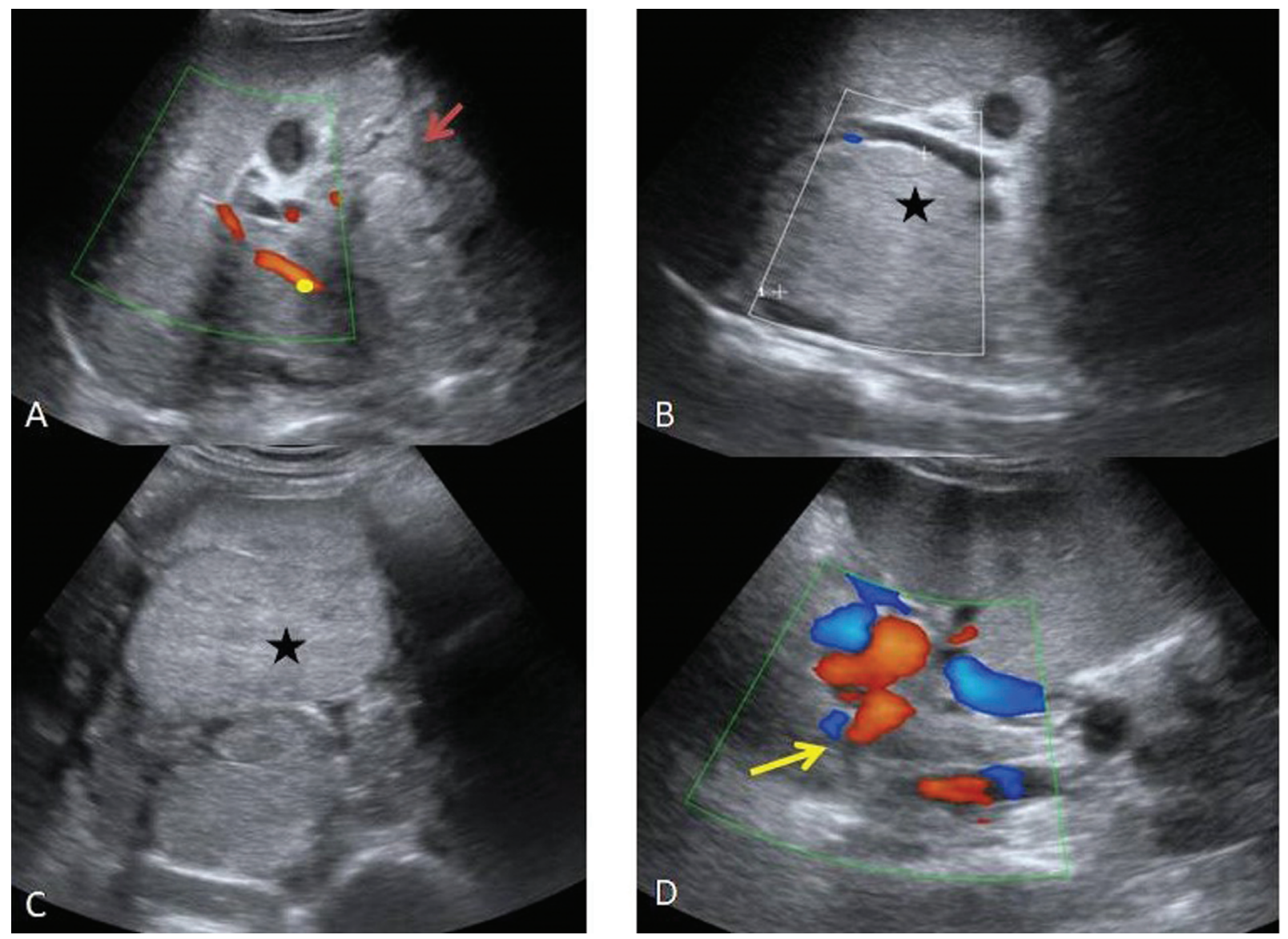

Fig. 1 (A-D) Ultrasound of abdomen showing a large lobulated isoechoic mass in the pancreatic head region (arrow) with intravenous extension and expansion of the main portal vein (asterisk) and its branches. Vascularity noted within the tumor thrombus (dot in A) with multiple periportal collaterals (arrow in D) also seen. 


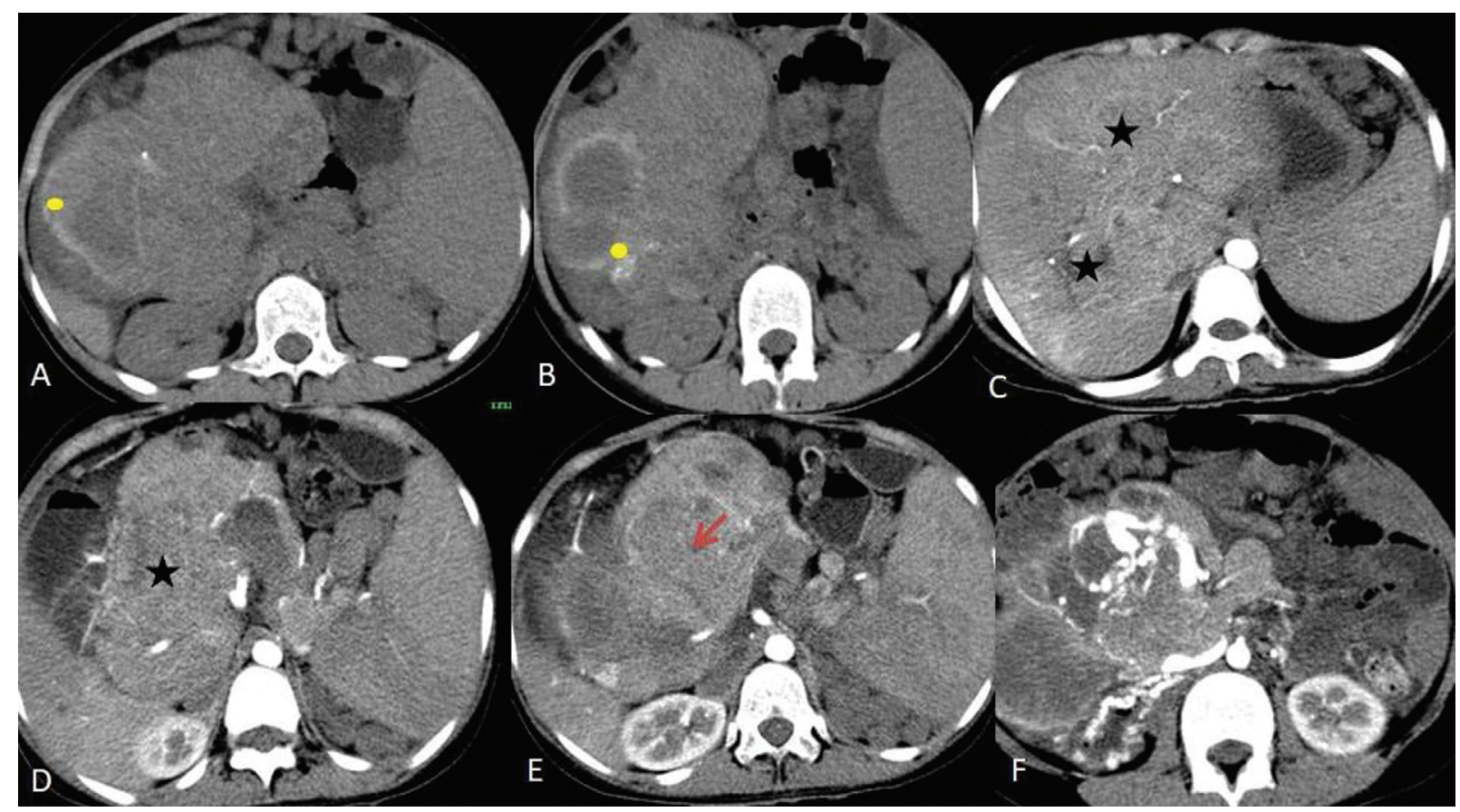

Fig. 2 (A, B) Contrast-enhanced computed tomography plain and (C-F) arterial phase axial sections showing peripheral curvilinear calcifications $(d o t)$ in the mass with heterogeneous enhancement in the pancreatic head region (arrows). Mass shows extension into the main portal vein and intrahepatic branches (asterisk).

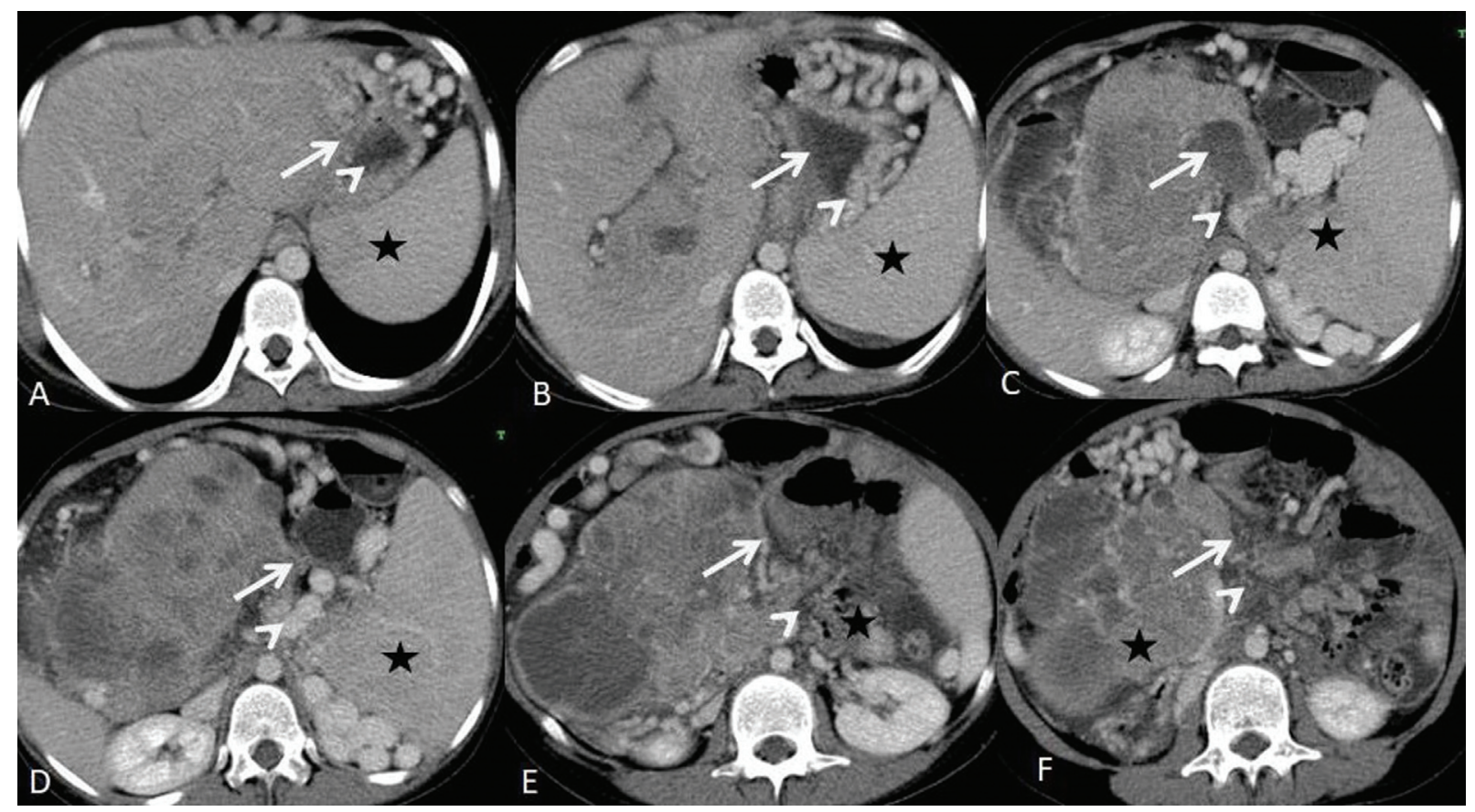

Fig. 3 (A-F) Contrast-enhanced computed tomography in hepatic venous phase scans showing large heterogeneous tumor (arrow) with splenomegaly (arrowhead) and collaterals in the peri-splenic and peritumoral region (asterisk).

multiple collaterals was seen. Whipple's pancreaticoduodenectomy was done, and intravascular thrombus was removed to the possible extent. The final histopathological diagnosis was SPN with foci of high-grade malignant transformation and lympho-vascular emboli with metastatic tumor in one peripancreatic lymph node. Immunohistochemistry showed positivity for synaptophysin and vimentin, and $\beta$-catenin confirming SPT (-Figs. 6 and 7).
The patient had postoperative collections, which was drained, developed septicemia and succumbed to the disease.

\section{Discussion}

SPT is an uncommon tumor and constitutes 1 to $2 \%$ of exocrine pancreatic tumors. Ninety percent of cases occur in females. SPT has a low-grade malignant potential and can 


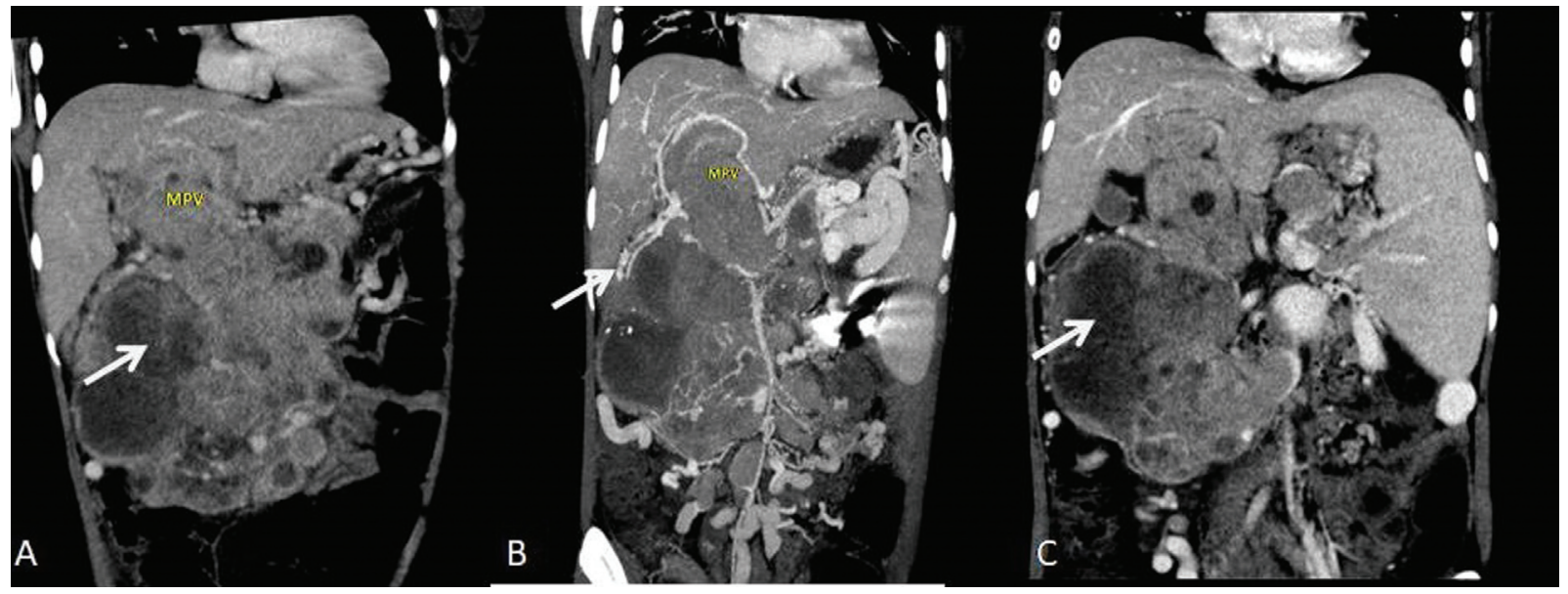

Fig. 4 (A-C) Coronal contrast-enhanced computed tomography in portal venous phase scans showing the large tumor (arrow) with splenomegaly, extension to the main portal vein with associated splenomegaly.

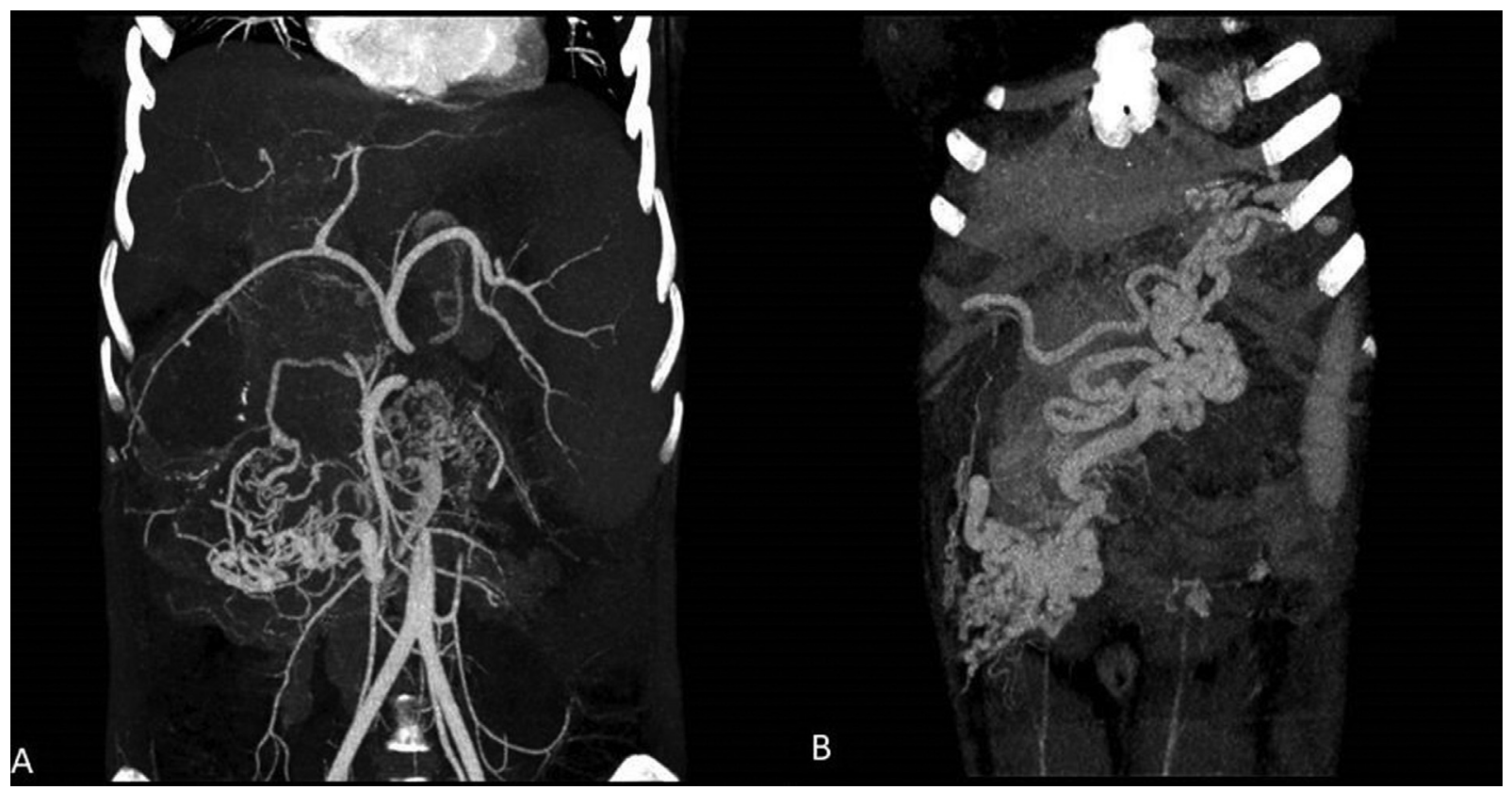

Fig. 5 (A) Contrast-enhanced computed tomography angiography and venography (B) with maximum intensity projection reconstruction showing tumor neovascularity from branches of superior mesenteric artery in the first image and spleno-portal collaterals due to portal hypertension in the second image.

be locally aggressive. ${ }^{2}$ According to the 2000 World Health Organization classification system, aggressive SPT should be classified as solid pseudopapillary carcinomas.

The tumor commonly involves the pancreatic head (34$40 \%$ ) or tail region (24-36\%). Large SPT will typically displace the adjacent structures rather than invading them. These tumors exhibit a soft consistency which rarely causes obstruction of the common bile duct or main pancreatic duct, even if located in the pancreatic head. ${ }^{6}$

Mean tumor size at presentation described is 6 to $8 \mathrm{~cm}$, though variable. Most of the tumors are moderate sized at presentation and present as palpable masses. About 15\% of the patients are asymptomatic. ${ }^{7}$
On ultrasound, the tumor could be predominantly solid, predominantly cystic, or mixed solid and cystic mass. Fineneedle aspiration is usually diagnostic., ${ }^{8,9}$

On cross-sectional imaging, SPT is encapsulated homogenous and enhancing when small and heterogeneous if large, due to hemorrhage and cystic degeneration. The fluid level has been reported in $18 \%$ of the cases. Peripheral calcification is seen in $30 \%$ of the cases. ${ }^{10}$ Central dystrophic calcifications may occur in areas of hemorrhage. Lamellar calcifications may also be present. ${ }^{11}$ If predominantly cystic, calcified and enhancing soft tissue components are seen in the periphery of the lesion. A study by Cantisani et $\mathrm{al}^{12}$ showed the most common enhancement pattern of SPT as early, peripheral, and heterogeneous enhancement during the arterial phase 


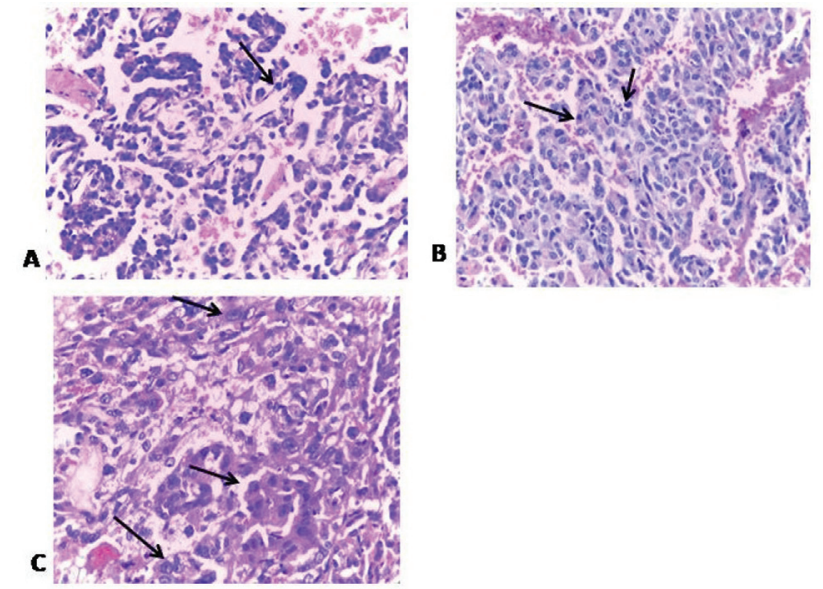

Fig. 6 (A-C) H\&E stain (magnification x400). Pseudopapillae (arrow in A) with vascular stalks surrounded by loosely cohesive fairly monomorphic tumor cells. Arrows in B and $\mathrm{C}$ show a tumor with highgrade areas showing increased nuclear atypia and mitosis. H\&E, hematoxylin and eosin.

with a progressive but heterogeneous filling of the lesion during the portal venous and equilibrium phases. The tumor capsule usually shows early and more intense enhancement.

Magnetic resonance imaging (MRI) has an added advantage over CT in the diagnosis of SPT as most of the tumors show evidence of hemorrhage with a variable signal on MRI. ${ }^{13} \mathrm{~A}$ hypointense rim suggestive of capsule can be seen on both $\mathrm{T} 1$ and T2 weighted images. CT has limitations in demonstrating hemorrhage, cystic degeneration, and capsule. ${ }^{14}$

Malignant transformation is rare and is suspected radiologically when the lesion is large with eccentric lobulated margin rather than smooth lobulation and when there is focal nodular or amorphous scattered calcification rather than rim calcification or if there is invasion into the surrounding tissue, or metastasis. ${ }^{15,16}$ The most common site of metastasis is the liver. Metastasis can also occur in the peritoneum, omentum, or lymph node. Solitary liver metastasis is not a contraindication to surgery.

The histogenesis of SPN is still enigmatic. This tumor may have its origin from the pluripotent stem cells of the genital ridges. This theory is strongly supported by features such as expression of progesterone and androgen receptors in the tumor, lack of expression of pancreatic epithelial markers, and regression of some tumors after menopause. The tumors are hormone dependent and may increase in size during pregnancy. Pathologically, although SPN was considered a "benign" or "borderline" tumor for many years, recent molecular evidence demonstrates alterations in cancer-associated genes and the ability to metastasize, and hence, this entity is now considered to be a low-grade malignant pancreatic tumor. ${ }^{17}$ The tumor shows a slow doubling time (765 days). ${ }^{13}$

SPN with foci of high-grade malignant transformation is an aggressive histologic subtype, the presence of nuclear atypia and high-mitotic rate may suggest an aggressive subtype. ${ }^{18}$

There is a lack of consensus regarding the utility of the $\mathrm{K}_{\mathrm{i}}-$ 67 index as a prognostic marker for SPT; however, Yang et al reported that a $\mathrm{K}_{\mathrm{i}}-67$ index $>4 \%$ might indicate a poor
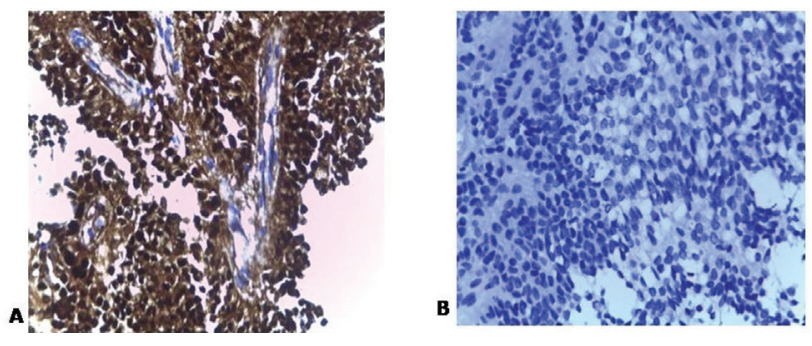

Fig. 7 (A, B) Immunohistochemistry showing positivity for $\beta$-catenin (both nuclei and cytoplasm) in A and negative for chromogranin in B (magnification $\mathrm{x} 400$ ).

prognosis in patients with SPT. ${ }^{19,20}$ Our patient had a $\mathrm{K}_{\mathrm{i}^{-}}$ 67 index of $3 \%$ but showed malignant transformation.

The literature search showed only a few cases of SPT with minimal intravascular extension. The key finding in our case is extensive intravascular tumor extension, not described so far. In spite of intravascular extension, the patient was taken for surgery, being the only treatment option available even in aggressive lesions. The role of chemotherapy and radiotherapy is not well established in invasive/malignant SPT.

Complete surgical resection is the treatment of choice in SPT. ${ }^{18}$ About 10 to $15 \%$ of patients have metastases at the time of diagnosis or develop metastases at some point in the future. The histopathology does not always completely and precisely predict the biological behavior of tumor, recurrence, or malignant potential. ${ }^{21}$ Hence, the importance of imaging and regular follow-up in all cases of SPT after surgical resection to diagnose early recurrence or distant metastasis should be emphasized.

\section{Conclusion}

A pancreatic mass in a young adult female with a heterogeneous appearance should raise suspicion for SPT. Malignant transformation should be suspected radiologically when there is angioinvasion or infiltration of adjacent structures as the role of the pathology of predicting malignant behavior is still uncertain. This is a unique case with extensive intravascular extension of thrombus not described in the literature so far.

\section{Funding}

None.

Conflict of Interest

None declared.

\section{References}

1 Rajendran AR. Pancreatic solid pseudo papillary epithelial neoplasm (Spen)-a case report. University Journal of Medicine and Medical Specialities 2018;4(05):

2 Mujtahedi SS, Shetty SK, Lobo FD. Solid pseudopapillary epithelial neoplasm (SPEN) of the pancreas involving the distal body and proximal tail: a case report. Int J Surg Case Rep 2021; $80: 105519$ 
3 Ilaiah M, Mallikarjun P, Mujeeb FA. Solid pseudopapillary epithelial neoplasm (spen) of pancreas: a case report and review of literature. Indian J Appl Res 2015;V(X):86-88

4 Sunkara S, Williams TR, Myers DT, Kryvenko ON. Solid pseudopapillary tumours of the pancreas: spectrum of imaging findings with histopathological correlation. Br J Radiol 2012;85(1019): e1140-e1144

5 Chang H, Gong Y, Xu J, Su Z, Qin C, Zhang Z. Clinical strategy for the management of solid pseudopapillary tumor of the pancreas: aggressive or less? Int J Med Sci 2010;7(05):309-313

6 Bollen TL, Wessels FJ. Radiological workup of cystic neoplasms of the pancreas. Visc Med 2018;34(03):182-190

7 Dinarvand P, Lai J. Solid pseudopapillary neoplasm of the pancreas: a rare entity with unique features. Arch Pathol Lab Med 2017; 141(07):990-995

8 Chon HK, Choi KH, Kim TH. An unusual presentation of a solid pseudopapillary tumor of the pancreas mimicking adenocarcinoma. Clin Endosc 2020;53(05):615-619

9 Kucera JN, Kucera S, Perrin SD, Caracciolo JT, Schmulewitz N, Kedar RP. Cystic lesions of the pancreas: radiologic-endosonographic correlation. Radiographics 2012;32(07):E283-E301

10 Buetow PC, Buck JL, Pantongrag-Brown L, Beck KG, Ros PR, Adair CF. Solid and papillary epithelial neoplasm of the pancreas: imaging-pathologic correlation on 56 cases. Radiology 1996; 199(03):707-711

11 Sarno A, Tedesco G, De Robertis R, Marchegiani G, Salvia R, D'Onofrio M. Pancreatic cystic neoplasm diagnosis: role of imaging. Endosc Ultrasound 2018;7(05):297-300

12 Cantisani V, Mortele KJ, Levy A, et al. MR imaging features of solid pseudopapillary tumor of the pancreas in adult and pediatric patients. AJR Am J Roentgenol 2003;181(02):395-401
13 Choi JY, Kim MJ, Kim JH, et al. Solid pseudopapillary tumor of the pancreas: typical and atypical manifestations. AJR Am J Roentgenol 2006;187(02):W178-W186

14 Antoniou EA, Damaskos C, Garmpis N, et al. Solid pseudopapillary tumor of the pancreas: a single-center experience and review of the literature. In Vivo 2017;31(04):501-510

15 Lee JH, Yu JS, Kim H, et al. Solid pseudopapillary carcinoma of the pancreas: differentiation from benign solid pseudopapillary tumour using CT and MRI. Clin Radiol 2008;63(09): 1006-1014

16 Ramakrishnan KK, Varma S, Govindaju NK, Rajan SC. Polarising Frantz tumors-benign and malignant solid pseudopapillary epithelial neoplasm of pancreas-twin case reports. 2021;23(09):

17 La Rosa S, Bongiovanni M. Pancreatic solid pseudopapillary neoplasm: key pathologic and genetic features. Arch Pathol Lab Med 2020;144(07):829-837

18 Tanapanpanit O, Kanngurn S, Pongpirul K. Frantz's tumour, solid pseudopapillary epithelial neoplasm (SPEN). BMJ Case Rep 2016; 2016:1-2

19 Yang F, Yu X, Bao Y, Du Z, Jin C, Fu D. Prognostic value of Ki-67 in solid pseudopapillary tumor of the pancreas: Huashan experience and systematic review of the literature. Surgery 2016;159 (04):1023-1031

20 Anila KARN, Nayak N, Muralee M, Venugopal BP, Mony RP. Solidpseudopapillary neoplasm of the pancreas: a classical presentation with unique paranuclear dot like immunostaining with $C D$ 99. Downloaded Free. Available at: http//www.ijpmonline.org. Accessed Oct 3, 2021, IP 4937182153.

21 Zalatnai A, Kis-Orha V. Solid-pseudopapillary neoplasms of the pancreas is still an enigma: a clinicopathological review. Pathol Oncol Res 2020;26(02):641-649 